\title{
Medical Comanagement of Hip Fracture Patients Is Not Associated with Superior Perioperative Outcomes: A Propensity Score-Matched Retrospective Cohort Analysis of the National Surgical Quality Improvement Project
}

\author{
Bryan G Maxwell, MD, MPH' ${ }^{1 *}$ Amer Mirza, MD²
}

'Department of Anesthesiology, Legacy Emanuel Medical Center, Portland, Oregon; ${ }^{2}$ Department of Surgery, Legacy Emanuel Medical Center, Portland, Oregon.

BACKGROUND: Medical comanagement entails a significant commitment of clinical resources with the aim of improving perioperative outcomes for patients admitted with hip fractures. To our knowledge, no national analyses have demonstrated whether patients benefit from this practice.

METHODS: We performed a retrospective cohort analysis of the American College of Surgeons (ACS) National Surgical Quality Improvement Program (NSQIP) targeted user file for hip fracture 2016-2017. Medical comanagement is a dedicated variable in the NSQIP. Propensity score matching was performed to control for baseline differences associated with comanagement. Matched pairs binary logistic regression was then performed to determine the effect of comanagement on the following primary outcomes: mortality and a composite endpoint of major morbidity.

RESULTS: Unadjusted analyses demonstrated that patients receiving medical comanagement were older and sicker with a greater burden of comorbidities. Comanagement did not have a higher proportion of patients participating in a standardized hip fracture program (53.6\% vs $53.7 \% ; P>.05)$. Comanagement was associated with a higher unadjusted rate of mortality (6.9\% vs $4.0 \%$, odds ratio [OR] 1.79: 1.44-2.22; $P<.0001)$ and morbidity (19.5\% vs $9.6 \%$, OR 2.28: $1.98-2.63$; $P<.0001)$. After propensity score matching was used to control for baseline differences associated with comanagement, patients in the comanagement cohort continued to demonstrate inferior mortality (OR 1.36: 1.02-1.81; $P=.033$ ) and morbidity (OR 1.82: 1.52-2.20; $P<.0001)$.

CONCLUSIONS: This analysis does not provide evidence that dedicated medical comanagement of hip fracture patients is associated with superior perioperative outcomes. Further efforts may be needed to refine opportunities to modify the significant morbidity and mortality that persists in this population. Journal of Hospital Medicine 2020;15:468-474. (c) 2020 Society of Hospital Medicine rate ip fractures are a large source of morbidity and mortality in the United States, with $>1.5$ million patients affected every year. ${ }^{1}$ These patients are primarily older adults with a significant burden of associated medical comorbidities. ${ }^{2}$ The outcomes of nonoperative management are poor with regard to mortality, ${ }^{3}$ although operative management of hip fractures remains associated with a high rate of morbidity and mortality compared with several other surgical procedures, substantial resources remain devoted to the operative repair of hip fractures and to process improvement strategies for perioperative care.

Medical comanagement involves having a second nonsurgical primary team-often an internist, a hospitalist, a geriatrician, or an anesthesiologist—who would follow the patient

*Corresponding Author: Bryan G Maxwell, MD, MPH; Email: bryanmaxwell@ gmail.com; Telephone: 503-413-4112.

Published online first December 18, 2019.

Received: July 17, 2019; Revised: September 25, 2019;

Accepted: October 7, 2019

๑ 2019 Society of Hospital Medicine DOI 10.12788/jhm.3343 during the hip fracture admission, and provide daily care directed toward both the hip fracture and its associated management challenges and the patient's underlying comorbidities. This includes taking a primary or shared role in daily rounding, writing progress notes, writing orders, managing medications and therapies, disposition planning, and discharge. One argument for this practice has centered around an efficiency proposition for surgeons to spend more of their time operating and less time in these tasks of acute care management. The primary argument, though, for medical comanagement has been an outcomes proposition that frail, elderly patients with significant medical comorbidities benefit from a nonsurgeon's focused attention to their coexisting medical problems and the interaction with the surgical issues posed by operative intervention for hip fracture. A number of previous studies have demonstrated an association between comanagement and improved perioperative outcomes. ${ }^{4,5}$ However, the most convincing improvements in several studies have been process indicators (eg, time from admission to surgery, length of stay, nurse/surgeon satisfaction) without significant differences in mortality or major morbidity. ${ }^{6-8}$ Several studies were methodologically limited due to the use of historical controls, 9,10 and 
several were conducted in focused clinical settings (eg, a single tertiary academic center), leaving uncertainty about external validity for other care environments. ${ }^{6,7}$ To our knowledge, comanagement has not been examined in the American College of Surgeons (ACS) National Surgical Quality Improvement Program (NSQIP) dataset of hip fracture patients.

The NSQIP database offers a unique tool for clinical outcomes research because its variables are prospectively collected by a trained clinical reviewer at each participating site. Data are deidentified and aggregated into a national database, which has grown from 121 participating sites in 2005 to 708 participating sites in 2017 and now contains data on more than 6.6 million patients. The targeted hip fracture participant use file (PUF) adds additional variables and is available beginning with 2016. Internal audits ensure a high level of data reliability. ${ }^{11}$ The NSOIP has compared favorably with single-institution morbidity and mortality conference systems, ${ }^{12}$ multi-institution clinical databases, ${ }^{13}$ and administrative databases ${ }^{14}$ in accurately capturing 30-day outcomes. Unlike other databases, outcomes are recorded within 30 days even if they occur after the initial postoperative discharge. Comanagement is a dedicated variable in the NSOIP hip fracture dataset.

This study sought to examine the effect of medical comanagement on perioperative outcomes in this contemporary NSQIP database.

\section{METHODS}

This study was exempt from the Institutional Review Board review because it uses deidentified data.

We used the targeted hip fracture NSQIP PUF for 2016-2017 to examine perioperative outcomes among patients undergoing hip fracture repair and assess the relationship with medical comanagement, which is a dedicated variable in the NSOIP hip fracture database. We included patients in the comanagement cohort if they received comanagement for part or all of their hip fracture hospitalization.

Demographic, comorbidity, and preoperative variables were examined between the two cohorts. Hypoalbuminemia, as a marker of malnutrition and frailty, was defined as a preoperative serum albumin level $<3.5 \mathrm{~g} / \mathrm{dL}$, which has demonstrated independent predictive value for adverse outcomes in hip fracture patients in the NSQIP. ${ }^{15,16}$ Predicted morbidity and mortality rates are calculated as probabilities available for each patient in the PUF based on a NSOIP hierarchical regression analysis of patient-level factors to predict outcomes (eg, not including hospital or provider factors). We also examined the relationship in regard to participation in a standardized hip fracture program (SHFP), which is a multidisciplinary protocolized pathway for hip fracture patients that may include order sets, structured care coordination, involvement of multidisciplinary therapy personnel, and daily milestones and discharge criteria. Participation in an SHFP is recorded in the NSOIP and has demonstrated an association with significantly improved outcomes in this same dataset, the targeted hip fracture PUF. ${ }^{17}$

Logistic regression was performed using all baseline variables identified to be significantly different between the co- horts, as well as the following variables with a priori potential importance in predicting membership in the comanagement cohort: admission year, sex, American Society of Anesthesiologists (ASA) physical status $\geq 4$, and participation in an SHFP. Propensity scores were calculated using the significant variables from this model (Table 1) and the abovementioned a priori potential confounders, and then propensity score matching was performed using a greedy matching algorithm (matching ratio $1: 1$, caliper width $=0.1$ pooled standard deviations of the logit of the propensity score) to create comanagement and control cohorts for matched analysis.

The primary outcomes were 30-day mortality and a composite endpoint of major morbidity, including readmission, pulmonary complications (pneumonia, reintubation, prolonged mechanical ventilation, and pulmonary embolism [PE]), septic shock, stroke, myocardial infarction, cardiac arrest, or death. Secondary outcomes included postoperative length of stay, disposition at postoperative day 30 , and process compliance measures (proportion of patients allowed to be weight-bearing as tolerated on postoperative day 1 , and proportion of patients appropriately prescribed deep venous thrombosis [DVT] prophylaxis for 28 days, proportion of patients appropriately prescribed bone protective medication [eg, vitamin $D$, bisphosphonates, teriparatide, denosumab, and raloxifene] postoperatively).

Descriptive variables are reported as median (interquartile range) and number (percentage), unless otherwise noted. Continuous outcomes were compared using a Mann-Whitney-Wilcoxon test. Binary outcomes were compared using Fisher's exact tests (or a Pearson's Chi-square for more than two response levels) and odds ratios with $95 \%$ confidence intervals. Matched pairs binary logistic regression was used to examine the relationship between comanagement and the primary outcomes of mortality and morbidity in the propensity score-matched cohorts, as well as between comanagement and secondary outcomes. Friedman's test was used for a secondary outcome with more than two response levels (disposition at 30 days). Analyses were performed using SAS (SAS 9.4; SAS Institute, Cary, North Carolina) with a predetermined alpha value of 0.05 to determine statistical significance.

\section{RESULTS}

A total of 19,896 Hip fracture patients were categorized into a medical comanagement cohort of 17,600 (88.5\%) patients and a cohort without comanagement of 2,296 patients (11.5\%). Baseline characteristics of the two unadjusted cohorts before propensity score matching are presented in Table 2.

Patients in the comanagement cohort were older and sicker in terms of almost every comorbidity and condition evaluated (Table 2). These differences were also reflected in a higher predicted mortality by the NSOIP hierarchical regression-based equations for mortality (3.5\% [1.7\%-7.0\%] vs 2.5\% [0.9\%-6.1\%], $P<.0001)$ and morbidity $(9.1 \%[6.9 \%-12.5 \%]$ vs $8.5 \%[6.1 \%-$ $12.1 \%], P \leq .0001)$. As predicted, the observed, unadjusted rate of death in the comanagement cohort was higher than that in the cohort without comanagement: $\mathrm{n}=1,210(6.9 \%)$ vs $\mathrm{n}=91$ (4.0\%), odds ratio (OR) 1.79: 1.44-2.22; $P<.0001$, as was the 
TABLE 1. Variables Identified in Logistic Regression to be Used in Propensity Score

\begin{tabular}{|c|c|c|c|}
\hline & OR & $95 \% \mathrm{Cl}$ & $P$ Value \\
\hline Age (years) & 1.02 & $(1.02-1.03)$ & $<.0001$ \\
\hline Male & 0.87 & $(0.78-0.97)$ & .010 \\
\hline Caucasian, non-Hispanic & 0.10 & $(0.09-0.11)$ & $<.0001$ \\
\hline Chronic obstructive pulmonary disease & 0.72 & $(0.59-0.87)$ & .0006 \\
\hline Hypertension requiring medication & 0.69 & $(0.62-0.76)$ & $<.0001$ \\
\hline Diabetes requiring medication & 0.60 & $(0.52-0.69)$ & $<.0001$ \\
\hline Baseline heart failure & 0.40 & $(0.26-0.62)$ & $<.0001$ \\
\hline Obese & 0.97 & $(0.84-1.13)$ & .72 \\
\hline Independent baseline functional status & 1.12 & $(1.01-1.24)$ & .032 \\
\hline Admitted for $>48$ hours prior to surgery & 0.48 & $(0.39-0.60)$ & $<.0001$ \\
\hline Preoperative delirium & 0.50 & $(0.41-0.61)$ & $<.0001$ \\
\hline Preoperative blood transfusion & 0.55 & $(0.41-0.74)$ & $<.0001$ \\
\hline Preoperative renal failure & 0.79 & $(0.68-0.92)$ & .002 \\
\hline Preoperative coagulopathy & 0.80 & $(0.68-0.95)$ & .009 \\
\hline
\end{tabular}

Abbreviations: ASA, American Society of Anesthesiologists; OR, odds ratio.

unadjusted rate of the composite endpoint of major morbidity: $n=3,425$ (19.5\%) vs $n=220$ (9.6\%), OR 2.28: 1.98-2.63, $P<$ .0001 . There was no difference in the prevalence of using an SHFP in the comanagement and noncomanagement cohorts ( $n=9,441,53.6 \%$ vs $n=1,232,53.7 \%, P>.05$ ).

Logistic regression modeling of the probability of membership in the comanagement cohort yielded satisfactory results (convergent model, null hypothesis rejected, area under the curve of the model receiver operating curve $[A U R O C]=0.81$ ). Propensity scores were calculated using the significant variables from this model, as detailed in Table 1. Propensity score matching was then performed with excellent results as follows: $n=2,278$ of 2,296 (99.2\%) potential pairs were successfully matched, residual absolute standardized difference $=0.0039$ (99.7\% reduction), variance ratio $=1.01$. This satisfies the traditional criterion for a satisfactory variable balance in propensity score matching of a standardized difference $\leq 0.25$ and a variance ratio between 0.5 and 2.0. It is also worthy of note that the propensity score matching process successfully eliminated the baseline difference in the NSQIP-predicted probability of mortality $(2.7 \%[1.1 \%-5.8 \%]$ vs $2.5 \%[0.9 \%-6.2 \%], P=.15)$ and morbidity (8.6\% [6.5\%-11.7\%] vs $8.6 \%$ [6.1\%-12.2\%], $P=.80$ ).

The characteristics of the propensity score-matched cohorts $(n=2,278$ each) are shown in Table 3. Matching resulted in a satisfactory balance of measurable covariates between the two cohorts, with the exception of small (but statistically significant) differences in the prevalence of hypoalbuminemia and the distribution of fracture type.

The comanagement cohort did not experience superior results for either of the two primary outcomes mortality (OR 1.36: $1.02-1.81 ; P=.033$ ) or in the composite endpoint of morbidity (OR 1.82: 1.52-2.20; $P<.0001$ ). The secondary outcomes of the two cohorts of patients are shown in Table 4. The comanagement cohort did not have superior outcomes in any variable examined, except for a slightly higher proportion of patients who were appropriately prescribed DVT prophylaxis. Despite the prophylaxis, the comanagement cohort did not have a smaller proportion of patients who experienced a DVT or PE.

Post hoc subgroup analysis was performed to assess whether comanagement demonstrated an association with improved 
TABLE 2. Baseline Characteristics of the Unadjusted Comanagement and Control Cohorts

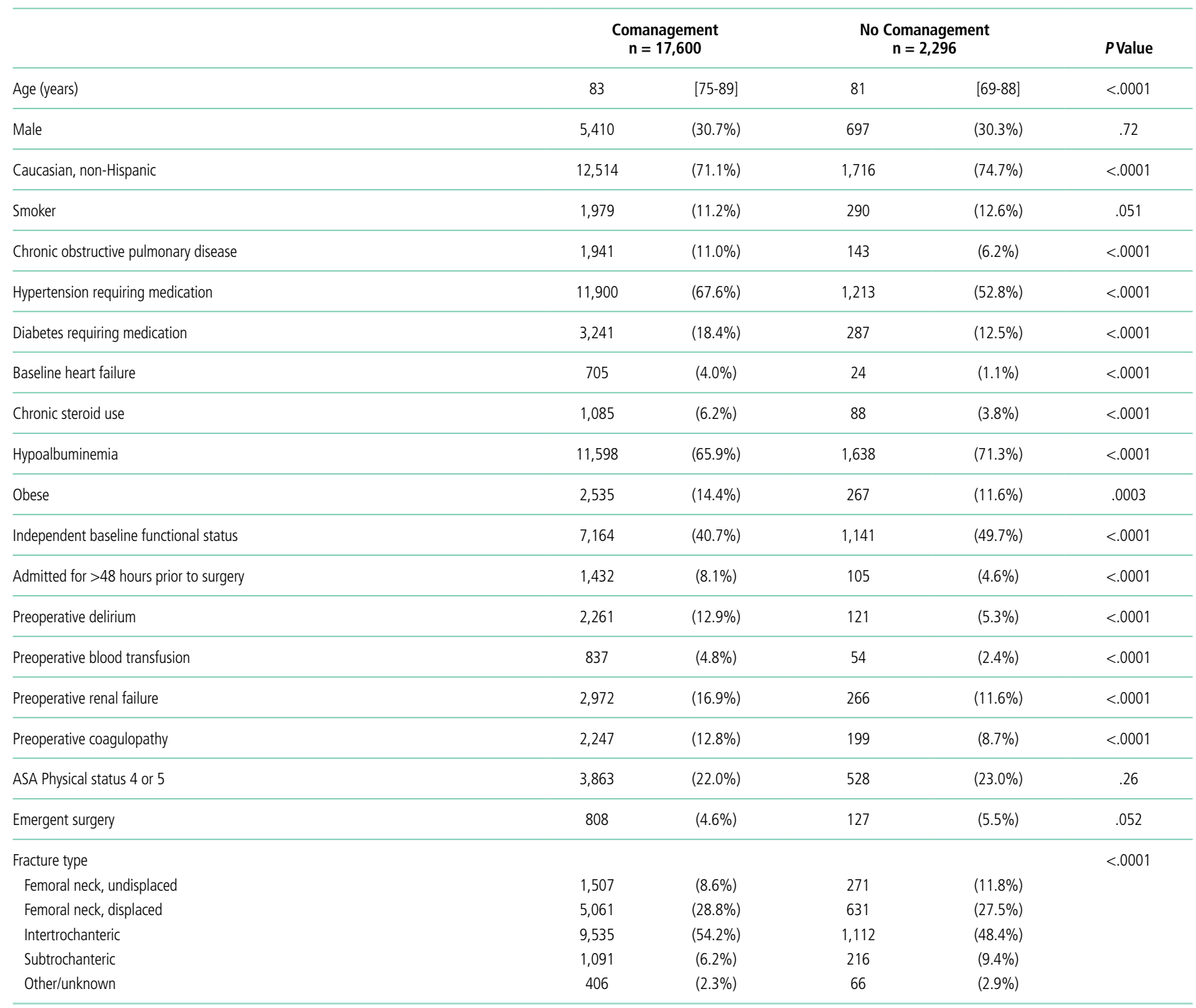

Values are median [interquartile range] or number (percentage) as appropriate

Abbreviation: ASA, American Society of Anesthesiologists

outcomes depending on whether patients were or were not treated in an SHFP. This stratified analysis produced the same results as the primary analysis; ie, comanagement was not associated with improved outcomes in either subgroup.

\section{DISCUSSION}

The primary finding of this study is that even once propensity score matching eliminated nearly all discernible baseline differences between the cohorts of hip fracture patients with and without medical comanagement during their hospitalization, and comanagement was not associated with superior (and in fact was associated with still inferior) perioperative outcomes.

As is evident from the baseline differences shown in Table 2 , medical comanagement is utilized in a patient population that has significant comorbidities and adverse patient factors.
The NSQIP provides a robust opportunity to remove the effects of these confounding variables because of the richness of variables in the dataset. For instance, some studies used a summary score for patient frailty, which has been an apparent predictor of worse clinical outcomes in this population. ${ }^{18,19}$ The NSQIP analyzes each component of the frailty score (diabetic status, history of COPD or current pneumonia, congestive heart failure, hypertension requiring medication, and nonindependent functional status) as well as to add additional variables (eg, low serum albumin level) and propensity score matching on each of these variables individually.

It is also important to note that although prior analyses have demonstrated that SHFPs are associated with better outcomes in this database, ${ }^{17}$ comanagement did not correlate with the use of an SHFP, nor did comanagement demonstrate any asso- 
TABLE 3. Baseline Characteristics of the Propensity Score-Matched Cohorts

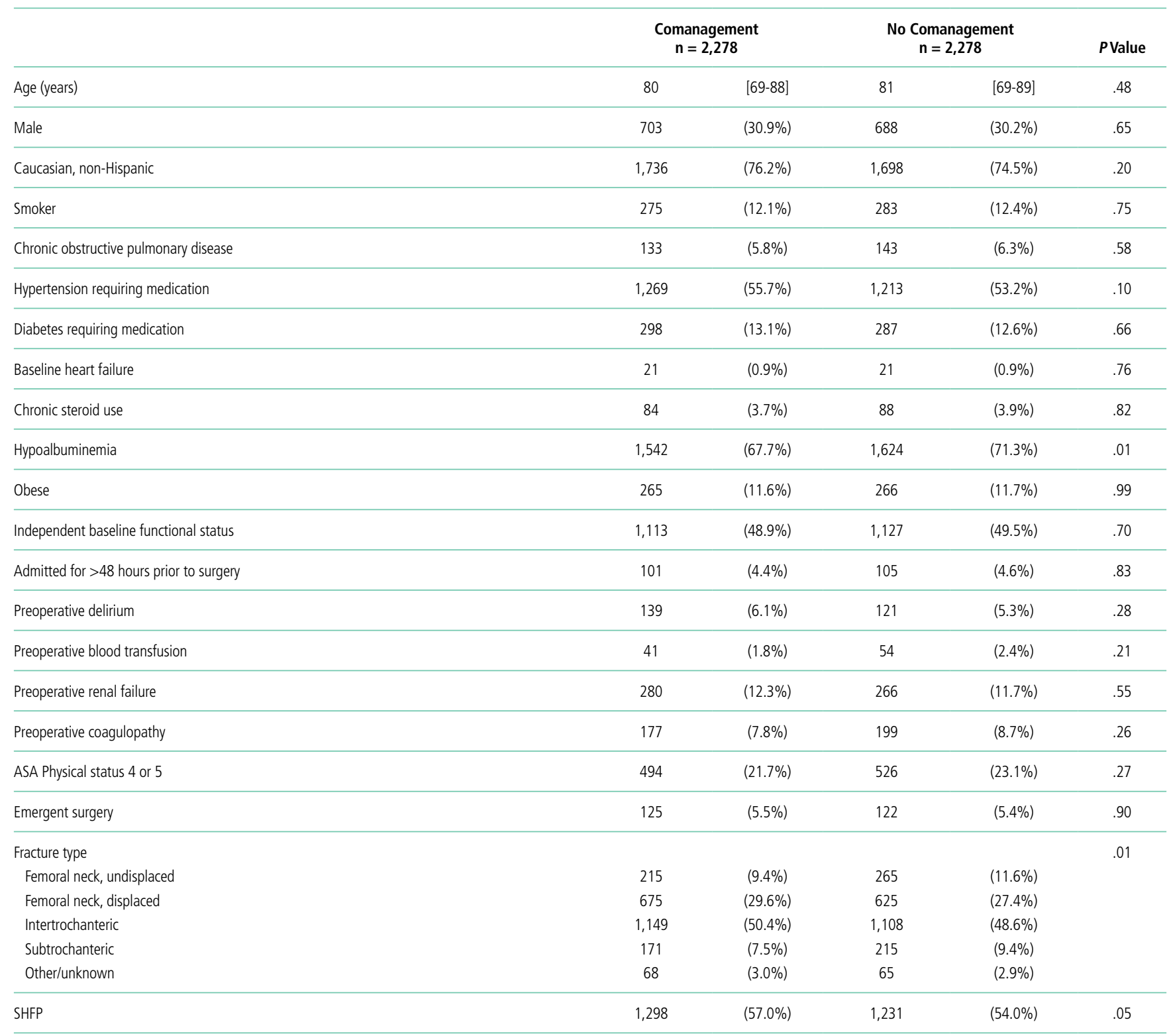

Values are median [interquartile range] or number (percentage) as appropriate.

Abbreviations: ASA: American Society of Anesthesiologists; SHFP, standardized hip fracture program.

ciation with better outcomes in the subgroup who participated in an SHFP or in the subgroup who did not.

This retrospective cohort analysis cannot, of course, demonstrate causation. Several limitations are worth noting. The ability to use any retrospective dataset depends on the quality of the variable definitions and the data quality contained in it. Although the NSQIP has demonstrated high validity and interobserver variability compared with other data sources, some imperfections and heterogeneity (for instance, in the way two different institutions may define comanagement) may be present.

It is important to note that any propensity score-matched analysis incurs the risk of residual/unmeasured confounding, since the power of this technique still depends on the presence of measured variables to match, and no match is ever perfect. For instance, some variables remain imperfectly balanced in the matched cohorts (eg, hypoalbuminemia and fracture type, Table 3). These differences may reach statistical significance because of large sample size without obvious clinical significance, but they illustrate the point that residual confounding may persist. It is also possible that some detection bias is present in the comanagement cohort, if dedicated comanagement personnel are more likely to diagnose complications (eg, pneumonia, PE) that require some clinical suspicion to be identified. We doubt that this plays a dominant role, for the NSOIP is relatively robust to this potential bias because of 
TABLE 4. Outcomes in the Propensity Score-Matched Cohorts

\begin{tabular}{|c|c|c|c|c|c|c|c|}
\hline \multirow[b]{2}{*}{ Mortality } & \multicolumn{2}{|c|}{$\begin{array}{c}\text { Comanagement } \\
n=2,278\end{array}$} & \multicolumn{2}{|c|}{$\begin{array}{l}\text { No Comanagement } \\
n=2,278\end{array}$} & \multirow{2}{*}{$\begin{array}{c}\mathrm{OR} \\
1.36\end{array}$} & \multirow{2}{*}{$\begin{array}{c}95 \% \mathrm{Cl} \\
(1.02,1.81)\end{array}$} & \multirow{2}{*}{$\begin{array}{r}\text { PValue } \\
.033\end{array}$} \\
\hline & 120 & & 91 & & & & \\
\hline Unplanned readmission & 141 & $(6.2 \%)$ & 94 & $(4.1 \%)$ & & & \\
\hline Pneumonia & 86 & $(3.8 \%)$ & 43 & $(1.9 \%)$ & & & \\
\hline Myocardial infarction & 67 & $(2.9 \%)$ & 15 & $(0.7 \%)$ & & & \\
\hline Septic shock & 28 & $(1.2 \%)$ & 19 & $(0.8 \%)$ & & & \\
\hline Cardiac arrest & 9 & $(0.4 \%)$ & 8 & $(0.4 \%)$ & & & \\
\hline Renal failure & 16 & $(0.7 \%)$ & 6 & $(0.3 \%)$ & & & \\
\hline $\begin{array}{l}\text { Prolonged mechanical ventilation or unplanned } \\
\text { reintubation }\end{array}$ & 23 & $(1.0 \%)$ & 10 & $(0.4 \%)$ & & & \\
\hline Prescription for DVT prophylaxis & 1,365 & $(59.9 \%)$ & 1,239 & $(54.4 \%)$ & 1.25 & $(1.11,1.41)$ & .0002 \\
\hline Weight-bearing on postoperative day 1 & 1,511 & $(66.3 \%)$ & 1,653 & $(72.6 \%)$ & 0.74 & $(0.66,0.85)$ & $<.0001$ \\
\hline Disposition at 30 days ${ }^{b}$ & & & & & & & $<.0001$ \\
\hline Home & 974 & $(50.7 \%)$ & 1090 & $(57.9 \%)$ & & & \\
\hline Still hospitalized & 118 & $(6.1 \%)$ & 78 & $(4.1 \%)$ & & & \\
\hline Subacute facility & 828 & $(43.1 \%)$ & 716 & $(38.0 \%)$ & & & \\
\hline
\end{tabular}

Values are median [interquartile range] or number (percentage) as appropriate

aindividual contributors to composite morbidity endpoint provided without $P$ values to avoid inappropriate multiple comparisons

bof patients alive and without missing data

Abbreviations: DVT, deep venous thrombosis; OR, odds ratio.

its rigorous process of relying on a trained clinical reviewer at each site (as opposed, for instance, to using billing codes), and several components of the composite morbidity endpoint (eg, reintubation, prolonged mechanical ventilation, stroke, cardiac arrest, or death) would be difficult to miss even if clinicians have low clinical suspicion or attentiveness. However, some potential remains.

It is also possible that comanagement is applied to sicker patients and functions more as a marker of that population than an intervention that improves results. To take a similar example, past literature has demonstrated a strong association between do-not-resuscitate (DNR) status and adverse outcomes. ${ }^{20-24}$ In all likelihood, the DNR status does not directly cause worse outcomes so much as it marks a sick and vulnerable population. Selection bias at the individual patient level may contribute to an association between comanagement and worse outcomes.

Similarly, institutions that routinely apply comanagement may care for a sicker patient population. To this end, institution-level variables may modulate the relationship between comanagement, SHFP participation, and outcomes. Comanagement and SHFP participation may cluster according to the surgeon, the institution, or the patient subtype (eg, ICU vs ward status). Unfortunately, individual hospital and surgeon identifiers are explicitly excluded from the publicly available
NSQIP PUF to protect program and patient confidentiality, so that advanced hierarchical modeling techniques cannot explore these relationships with this dataset.

Beyond these limitations, one plausible explanation for the lack of an association between comanagement and improved outcomes is that standardization and other continuous quality improvement processes have already accomplished a great deal, and the addition of comanagement of individual patients is not having an appreciably positive additional impact. Although the acuity and prevalence of comorbidities in the hip fracture population are high, many of their issues may be stereotyped enough that thoughtful, well-designed algorithms and protocols may serve them nearly as well, if not better than individual comanagement.

This admittedly speculative explanation has significant implications for resource utilization and patient care. Medical comanagement involves a heavy investment of time, energy, and money on the part of a second medical team to deliberately duplicate some aspects of daily care with the intended goal of improving patient outcomes. The results of this study may provide motivation for efforts to hybridize or modify the involvement of comanaging physicians and teams-for instance, to guide and refine the creation and revision of SHFP protocols without providing daily comanagement to each individual patient and/or to implement more iterative, continuous process improvement 
initiatives. ${ }^{25}$ Our results may also help direct healthcare systems to focus elsewhere in the search for modifiable process and care delivery variables that can move the needle on the significant morbidity and mortality that still exist in this population.

Disclosures: The authors have nothing to disclose.

Funding: Intramural support from Legacy Emanuel Medical Center relating to the LEMC trauma program.

\section{References}

1. Arneson TJ, Li S, Liu J, Kilpatrick RD, Newsome BB, St. Peter WL. Trends in hip fracture rates in US hemodialysis patients, 1993-2010. Am J Kidney Dis. 2013;62(4):747-754. https://doi.org/10.1053/j.ajkd.2013.02.368

2. Brauer CA, Coca-Perraillon M, Cutler DM, Rosen AB. Incidence and mortality of hip fractures in the United States. JAMA. 2009;302(14):1573-1579. https:// doi.org/10.1001/jama.2009.1462

3. Chlebeck JD, Birch CE, Blankstein M, Kristiansen T, Bartlett CS, Schottel PC. Nonoperative geriatric hip fracture treatment is associated with increased mortality. J Orthop Trauma. 2019;33(7):346-350. https://doi.org/10.1097/ BOT.0000000000001460

4. Wu X, Tian M, Zhang J, et al. The effect of a multidisciplinary co-management program for the older hip fracture patients in Beijing: a "pre- and post-" retrospective study. Arch Osteoporos. 2019;14(1):43. https://doi. org/10.1007/s11657-019-0594-1

5. Stephens JR, Chang JW, Liles EA, Adem M, Moore C. Impact of hospitalist vs. non-hospitalist services on length of stay and 30-day readmission rate in hip fracture patients. Hosp Pract. 2019;47(1):24-27. https://doi.org/10.1080/2 1548331.2019.1537850

6. Phy MP, Vanness DJ, Melton LJ, et al. Effects of a hospitalist model on elderly patients with hip fracture. Arch Intern Med. 2005;165(7):796-801. https://doi. org/10.1001/archinte.165.7.796

7. Batsis JA, Phy MP, Melton LJ, et al. Effects of a hospitalist care model on mortality of elderly patients with hip fractures. J Hosp Med. 2007;2(4):219225. https://doi.org/10.1002/jhm.207

8. Huddleston JM, Long KH, Naessens JM, et al. Medical and surgical comanagement after elective hip and knee arthroplasty: a randomized, controlled trial. Ann Intern Med. 2004;141(1):28-38. https://doi.org/10.7326/0003-4819141-1-200407060-00012

9. Gosch M, Hoffmann-Weltin Y, Roth T, Blauth M, Nicholas JA, Kammerlander C. Orthogeriatric co-management improves the outcome of long-term care residents with fragility fractures. Arch Orthop Trauma Surg. 2016;136(10):14031409. https://doi.org/10.1007/s00402-016-2543-4

10. Folbert EC, Hegeman JH, Vermeer M, et al. Improved 1-year mortality in elderly patients with a hip fracture following integrated orthogeriatric treatment. Osteoporos Int. 2017;28(1):269-277. https://doi.org/10.1007/s00198 016-3711-7

11. Shiloach M, Frencher SK, Steeger JE, et al. Toward robust information: data quality and inter-rater reliability in the American College of Surgeons National Surgical Quality Improvement Program. J Am Coll Surg. 2010;210(1):6-16 https://doi.org/10.1016/j.jamcollsurg.2009.09.031
12. Hutter MM, Rowell KS, Devaney LA, et al. Identification of surgical complications and deaths: an assessment of the traditional surgical morbidity and mortality conference compared with the American College of Surgeons-National Surgical Quality Improvement Program. J Am Coll Surg. 2006;203(5):618-624. https://doi.org/10.1016/j.jamcollsurg.2006.07.010

13. Davenport DL, Holsapple CW, Conigliaro J. Assessing surgical quality using administrative and clinical data sets: a direct comparison of the University HealthSystem Consortium Clinical Database and the National Surgical Quality Improvement Program data set. Am J Med Qual. 2009;24(5):395-402. https://doi.org/10.1177/1062860609339936

14. Yu P, Chang DC, Osen HB, Talamini MA. NSQIP reveals significant incidence of death following discharge. J Surg Res. 2011;170(2):e217-e224. https://doi. org/10.1016/j.jss.2011.05.040

15. Wilson J, Lunati M, Grabel Z, Staley C, Schwartz A, Schenker M. Hypoalbuminemia is an independent risk factor for 30-day mortality, postoperative complications, readmission, and reoperation in the operative lower extremity orthopedic trauma patient. J Orthop Trauma. 2019;33(6):284-291. https:// doi.org/10.1097/BOT.0000000000001448

16. Bohl DD, Shen MR, Hannon CP, Fillingham YA, Darrith B, Della Valle CJ. Serum albumin predicts survival and postoperative course following surgery for geriatric hip fracture. J Bone Jt Surg. 2017;99(24):2110-2118. https://doi. org/10.2106/JBJS.16.01620

17. Arshi A, Rezzadeh K, Stavrakis Al, Bukata S V, Zeegen EN. Standardized hospital-based care programs improve geriatric hip fracture outcomes: an analysis of the ACS-NSQIP targeted hip fracture series. J Orthop Trauma. 2019;33(6): e223-e228. https://doi.org/10.1097/BOT.0000000000001443

18. Traven SA, Reeves RA, Althoff AD, Slone HS, Walton ZJ. New 5-factor modified frailty index predicts morbidity and mortality in geriatric hip fractures. J Orthop Trauma. 2019;33(7):319-323. https://doi.org/10.1097/ BOT.0000000000001455

19. Wilson JM, Boissonneault AR, Schwartz AM, Staley CA, Schenker ML. Frailty and malnutrition are associated with inpatient post-operative complications and mortality in hip fracture patients. J Orthop Trauma. 2018;33(3):143-148. https://doi.org/10.1097/BOT.0000000000001386

20. Brovman EY, Pisansky AJ, Beverly A, Bader AM, Urman RD. Do Not Resuscitate Status as an independent risk factor for patients undergoing surgery for hip fracture. World J Orthop. 2017;8(12):902-912. https://doi.org/10.5312/ wjo.v8.i12.902

21. Brovman EY, Walsh EC, Burton BN, et al. Postoperative outcomes in patients with a do-not-resuscitate (DNR) order undergoing elective procedures. J Clin Anesth. 2018;48:81-88. https://doi.org/10.1016/j.jclinane.2018.05.007

22. Beverly A, Brovman EY, Urman RD. Comparison of postoperative outcomes in elderly patients with a do-not-resuscitate order undergoing elective and nonelective hip surgery. Geriatr Orthop Surg Rehabil. 2017;8(2):78-86. https://doi.org/10.1177/2151458516685826

23. Maxwell BG, Lobato RL, Cason MB, Wong JK. Perioperative morbidity and mortality of cardiothoracic surgery in patients with a do-not-resuscitate order. PeerJ. 2014;2013(1):1-10. https://doi.org/10.7717/peerj.245

24. Kazaure H, Roman S, Sosa JA. High mortality in surgical patients with do-not-resuscitate orders: analysis of 8256 patients. Arch Surg. 2011;146(8):922-928. https://doi.org/10.1001/archsurg.2011.69

25. Brañas F, Ruiz-Pinto $A$, Fernández $E$, et al. Beyond orthogeriatric co-management model: benefits of implementing a process management system for hip fracture. Arch Osteoporos. 2018;13(1):81. https://doi.org/10.1007/ s11657-018-0497-6 